\title{
BIFURCATIONS OF LIMIT CYCLES FROM CUBIC HAMILTONIAN SYSTEMS WITH A CENTER AND A HOMOCLINIC SADDLE-LOOP
}

\section{YUlin ZhaO AND Zhifen Zhang}

Abstract

It is proved in this paper that the maximum number of limit cycles of system

$$
\left\{\begin{array}{l}
\frac{d x}{d t}=y, \\
\frac{d y}{d t}=k x-(k+1) x^{2}+x^{3}+\epsilon\left(\alpha+\beta x+\gamma x^{2}\right) y
\end{array}\right.
$$

is equal to two in the finite plane, where $k>\frac{11+\sqrt{33}}{4}, 0<|\epsilon| \ll 1$, $|\alpha|+|\beta|+|\gamma| \neq 0$. This is partial answer to the seventh question in [2], posed by Arnold.

\section{Introduction}

Consider the Abelian integral

$$
I(h)=\oint_{\Gamma_{h}} Y(x, y) d x-X(x, y) d y, \quad h \in \Sigma,
$$

where $H(x, y), X(x, y)$ and $Y(x, y)$ are real polynomial of $x$ and $y, \Gamma_{h}$ is the compact component of $H(x, y)=h, \Sigma$ is the maximal interval of existence of $\Gamma_{h}$. Finding the lowest upper bound for the number of zeros of $I(h)$ is called the weakend Hilber-16th problem [1], which is closed related to determining the number of limit cycles of perturbed system

$$
\left\{\begin{array}{l}
\frac{d x}{d t}=\frac{\partial H}{\partial y}+\epsilon X(x, y), \\
\frac{d y}{d t}=-\frac{\partial H}{\partial x}+\epsilon Y(x, y),
\end{array}\right.
$$

where $0<|\epsilon| \ll 1$.

This work was done in 1995-1998, when the first author was a Ph.D. student in Peking University. 
In particular, suppose

$$
H(x, y)=\frac{1}{2} y^{2}+U(x)=h,
$$

where $U(x)$ is a real polynomial of $x$ with degree $n$. In this case, finding the number of zeros of $I(h)$ is one of the ten problems in $[\mathbf{2}]$. When $n=3$, this problem was investigated by many authors (e.g. [7], [8], [10], $[\mathbf{1 1}])$. When $n=4$, some results were given by $[\mathbf{5}],[\mathbf{1 2}],[\mathbf{1 3}],[\mathbf{1 6}],[\mathbf{1 7}]$, but this case is far from complete solving. In this paper, we study the case $n=4$ and the Hamiltonian vector field $d H=0$ possesses one center and one homoclinic saddle-loop, which has the following normal form

$$
\left\{\begin{array}{l}
\frac{d x}{d t}=y, \\
\frac{d y}{d t}=k x-(k+1) x^{2}+x^{3},
\end{array}\right.
$$

where $k>2$.

The system (1.4) has the first integral

$$
H(x, y)=\frac{1}{2} y^{2}-\frac{1}{2} k x^{2}+\frac{1}{3}(k+1) x^{3}-\frac{1}{4} x^{4}=h,
$$

and the phase portrait is shown in Figure 1.1. The closed ovals $\Gamma_{h}$ are defined for Hamiltonian values $h \in\left(\frac{-2 k+1}{12}, 0\right) . \quad H(x, y)=\frac{-2 k+1}{12}$ corresponds the center $(1,0), \Gamma_{0}=\left\{(x, y) \mid H(x, y)=0,0<x<x_{1}=\right.$ $\left.\frac{2(k+1)-\sqrt{2(k-2)(2 k-1)}}{3}\right\}$ corresponds the saddle point $(0,0)$ and homoclinic loop. The critical point $(k, 0)$ is a saddle.

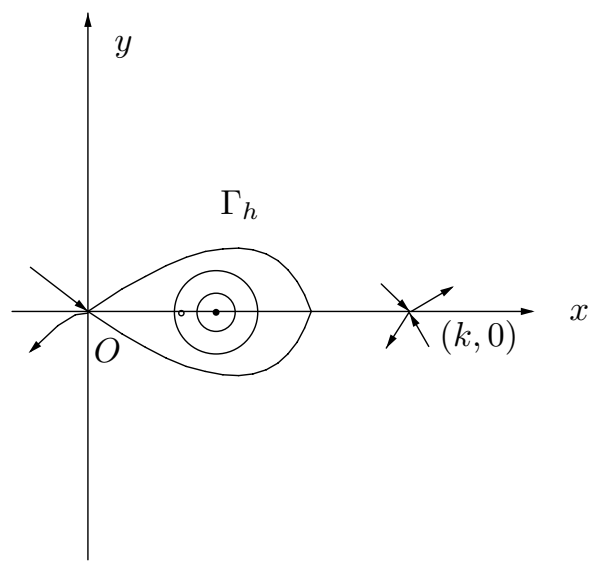

Figure 1.1 
Denote

$$
\begin{aligned}
I_{i}(h) & =\oint_{\Gamma_{h}} x^{i} y d x, \quad i=0,1,2, \\
I(h) & =\alpha I_{0}(h)+\beta I_{1}(h)+\gamma I_{2}(h),
\end{aligned}
$$

where the ovals $\Gamma_{h}, h \in\left(\frac{-2 k+1}{12}, 0\right)$, has negative (clockwise) orientation coinciding with the orientation of the vector field (1.4), $\alpha, \beta$ and $\gamma$ are arbitrary constants. The central result of this paper is the following theorem:

Theorem 1.1. The maximum number of limit cycles of the perturbed system

$$
\left\{\begin{array}{l}
\frac{d x}{d t}=y \\
\frac{d y}{d t}=k x-(k+1) x^{2}+x^{3}+\epsilon\left(\alpha+\beta x+\gamma x^{2}\right) y
\end{array}\right.
$$

is equal to two in the finite plane, where $k>\frac{11+\sqrt{33}}{4}, 0<|\epsilon| \ll 1$, $|\alpha|+|\beta|+|\gamma| \neq 0$.

Corollary 1.2. For $k>\frac{11+\sqrt{33}}{4}$, either $I(h)$ vanishes identically or its lowest upper bound of the number of zeros is equal to two, which is partial answer to the seventh problem in $[\mathbf{2}]$.

The paper is organized as follows: In section 2, Picard-Fuchs equation satisfied by $I_{0}(h), I_{1}(h)$ and $I_{2}(h)$ is derived and the expansions of $I(h)$ near its endpoints are given, the latter results reveal the connection between the Abelian integrals $I(h)$ and the limit cycles of system $(1.8)_{\epsilon}$ which tend to the center $(1,0)$ or homoclinic loop of $(1.4)$ as $\epsilon \rightarrow 0$. In section 3, instead of estimating the number of zeros of $I(h)$, we will prove that $I^{\prime \prime}(h)$ has at most two zeros, i.e., $I(h)$ has at most two inflection points in $\left(\frac{-2 k+1}{12}, 0\right)$, which implies the lowest upper bound of the number of zeros of $I(h)$ does not exceed three in the same interval. Using the fact $\omega(h)=\frac{I_{1}^{\prime \prime}(h)}{I_{0}^{\prime \prime}(h)}$ satisfies a Riccati equation, we get $\omega^{\prime}(h)>0$. Hence, the curve $\widetilde{\Omega}=\left\{(\omega, \nu) \mid \omega=\frac{I_{1}^{\prime \prime}(h)}{I_{0}^{\prime \prime}(h)}, \nu=\frac{I_{2}^{\prime \prime}(h)}{I_{0}^{\prime \prime}(h)}, h \in\left(\frac{-2 k+1}{12}, 0\right)\right\}$ can be defined. It is readily seen that the intersection points of line $\alpha+\beta \omega+$ $\gamma \nu=0$ with $\widetilde{\Omega}$ in $\omega \nu$-plane correspond the zeros of $I^{\prime \prime}(h)$, which shows that the convexity of $\widetilde{\Omega}$ determinates the number of the zeros of $I^{\prime \prime}(h)$. 
In section 4 , we make precise connection between the intersection points of $L: \alpha+\beta P+\gamma Q=0$ with the centroid curve $\Omega=\{(P, Q) \mid P=$ $\left.\frac{I_{1}(h)}{I_{0}(h)}, Q=\frac{I_{2}(h)}{I_{1}(h)}\right\}$ on one hand and the zeros of Abelian integral $I(h)$ on the other hand. Finally, the main results of this paper are proved in section 5. Some techniques in section 4 and section 5 are borrowed from $[4]$.

Remark. Unfortunately, the techniques we use in this present paper do not fit for the case of $2<k<\frac{11+\sqrt{33}}{4}$. Therefore, throughout this paper, we suppose $k>\frac{11+\sqrt{33}}{4}>4$ unless the opposite is claimed. Some computation in this paper is done by the computer program "Mathematica".

\section{Picard-Fuchs equation and the asymptotic expansions of $I(h)$ near its endpoints}

In this section we shall derive Picard-Fuchs equation satisfied by $I_{i}(h)$ and describe the behaviours of $I(h)$ near $h=0$ and $h=\frac{-2 k+1}{12}$.

Lemma 2.1. $I_{0}(h), I_{1}(h)$ and $I_{2}(h)$ satisfy the following Picard-Fuchs equation

$$
(4 h \mathbf{E}+\mathbf{S}) \mathbf{J}^{\prime}=\mathbf{N J}
$$

which is equivalent to

$$
G(h) \mathbf{J}^{\prime}=\mathbf{R J},
$$

where $\mathbf{E}$ is an unit matrix of order $3, \mathbf{J}=\operatorname{col}\left(I_{0}, I_{1}, I_{2}\right)$, and

$$
\begin{aligned}
\mathbf{S} & =\left(\begin{array}{ccc}
0 & \frac{1}{3} k(k+1) & \frac{1}{3}\left(-k^{2}+k-1\right) \\
0 & \frac{1}{3} k\left(k^{2}-k+1\right) & -\frac{1}{3}(k+1)(k-1)^{2} \\
0 & \frac{1}{3} k(k+1)(k-1)^{2} & \frac{1}{3}\left(-k^{4}+k^{3}+k^{2}+k-1\right)
\end{array}\right), \\
\mathbf{N} & =\left(\begin{array}{ccc}
3 & 0 & 0 \\
-\frac{1}{3}(k+1) & 4 & 0 \\
\frac{1}{3}\left(-k^{2}+k-1\right) & -\frac{2}{3}(k+1) & 5
\end{array}\right) \\
\mathbf{R} & =\left(\begin{array}{lll}
a_{00}(h) & a_{01}(h) & a_{02}(h) \\
a_{10}(h) & a_{11}(h) & a_{12}(h) \\
a_{20}(h) & a_{21}(h) & a_{22}(h)
\end{array}\right)
\end{aligned}
$$




$$
\begin{aligned}
G(h) & =192 h\left(h-\frac{-2 k+1}{12}\right)\left(h-\frac{k^{3}(k-2)}{12}\right), \\
a_{00}(h) & =144 h^{2}+\frac{4}{3}\left(-10 k^{4}+21 k^{3}-k^{2}+21 k-10\right) h \\
-\frac{4}{3} k^{3}(2 k-1)(k-2), & \\
a_{01}(h) & =-\frac{8}{3}(k+1)\left(k^{2}+5 k+1\right) h+\frac{14}{9} k^{2}(k+1)(2 k-1)(k-2), \\
a_{02}(h) & =20\left(k^{2}-k+1\right) h-\frac{5}{3} k^{2}(k-2)(2 k-1), \\
a_{10}(h) & =-16(k+1) h^{2}+\frac{4}{3} k(k+1)(2 k-1)(k-2) h, \\
a_{11}(h) & =192 h^{2}+\frac{8}{3}\left(-7 k^{4}+6 k^{3}+8 k^{2}+6 k-7\right) h, \\
a_{12}(h) & =20(k+1)(k-1)^{2} h, \\
a_{20}(h) & =16\left(-k^{2}+k-1\right) h^{2}+\frac{4}{3} k^{2}(k-2)(2 k-1) h, \\
a_{21}(h) & =-32(k+1) h^{2}-\frac{8}{3} k(k+1)\left(7 k^{2}-13 k+7\right) h, \\
a_{22}(h) & =240 h^{2}+20 k\left(k^{2}-k+1\right) h .
\end{aligned}
$$

Proof: It follows from (1.5) that

$$
\frac{\partial y}{\partial h}=\frac{1}{y}
$$

and

$$
y \frac{\partial y}{\partial x}=k x-(k+1) x^{2}+x^{3} .
$$

Obviously, (2.3) implies that

$$
I_{i}^{\prime}(h)=\oint_{\Gamma_{h}} \frac{x^{i}}{y} d x
$$

Mutiplying (2.4) by $y$ and integrating over $\Gamma_{h}$ give

$$
I_{3}=-k I_{1}+(k+1) I_{2} .
$$


Use (1.5) and (2.5) to get

$$
\begin{aligned}
I_{i}(h) & =\oint_{\Gamma_{h}} \frac{x^{i} y^{2}}{y} d x \\
& =\oint_{\Gamma_{h}} \frac{x^{i}\left(2 h+k x^{2}-\frac{2}{3}(k+1) x^{3}+\frac{1}{2} x^{4}\right)}{y} d x \\
& =2 h I_{i}^{\prime}+k I_{i+2}^{\prime}-\frac{2}{3}(k+1) I_{i+3}^{\prime}+\frac{1}{2} I_{i+4}^{\prime} .
\end{aligned}
$$

On the other hand, using (2.3), (2.4) and integrating by parts, we have

$$
\begin{aligned}
I_{i}(h) & =-\frac{1}{i+1} \oint_{\Gamma_{h}} x^{i+1} d y \\
& =-\frac{1}{i+1} \oint_{\Gamma_{h}} \frac{x^{i+1}\left(k x-(k+1) x^{2}+x^{3}\right)}{y} d x \\
& =-\frac{1}{i+1}\left(k I_{i+2}^{\prime}-(k+1) I_{i+3}^{\prime}+I_{i+4}^{\prime}\right) .
\end{aligned}
$$

Eliminating $I_{i+4}^{\prime}$ from (2.7) and (2.8) yields

$$
(i+3) I_{i}=4 h I_{i}^{\prime}+k I_{i+2}^{\prime}-\frac{1}{3}(k+1) I_{i+3}^{\prime} .
$$

This gives

$$
\begin{aligned}
& 3 I_{0}=4 h I_{0}^{\prime}+k I_{2}^{\prime}-\frac{1}{3}(k+1) I_{3}^{\prime}, \\
& 4 I_{1}=4 h I_{1}^{\prime}+k I_{3}^{\prime}-\frac{1}{3}(k+1) I_{4}^{\prime}, \\
& 5 I_{2}=4 h I_{2}^{\prime}+k I_{4}^{\prime}-\frac{1}{3}(k+1) I_{5}^{\prime} .
\end{aligned}
$$

Substituting (2.6) into (2.10), we obtain the first equation of (2.1). The formula (2.8) implies

$$
I_{i+4}^{\prime}=-(i+1) I_{i}(h)-k I_{i+2}^{\prime}+(k+1) I_{i+3}^{\prime} .
$$

Taking $i=0$ in (2.13) and using (2.6), the formula (2.11) give the second equation of (2.1).

Repeating the same arguments, we obtain the third equation. The lemma has been proved.

Denote

$$
P(h)=\frac{I_{1}(h)}{I_{0}(h)}, \quad Q(h)=\frac{I_{2}(h)}{I_{0}(h)},
$$

where $h \in\left[\frac{-2 k+1}{12}, 0\right]$. 
Proposition 2.2. $P(h), Q(h)$ are analytic function for $h \in\left[\frac{-2 k+1}{12}, 0\right)$, and

i)

$$
I_{i}\left(\frac{-2 k+1}{12}\right)=0, \quad I_{i}(h)>0, \quad i=0,1,2
$$

ii) $P\left(\frac{-2 k+1}{12}\right)=Q\left(\frac{-2 k+1}{12}\right)=1, \quad P(h)>0, \quad Q(h)>0$,

iii) $\quad P^{\prime}\left(\frac{-2 k+1}{12}\right)=-\frac{k-2}{2(k-1)^{2}}, \quad Q^{\prime}\left(\frac{-2 k+1}{12}\right)=-\frac{k-3}{2(k-1)^{2}}$,

iv)

$$
P^{\prime \prime}\left(\frac{-2 k+1}{12}\right)=\frac{(k-2)\left(-257+257 k-110 k^{2}\right)}{72(k-1)^{5}},
$$

$$
Q^{\prime \prime}\left(\frac{-2 k+1}{12}\right)=\frac{651-788 k+467 k^{2}-110 k^{3}}{72(k-1)^{5}} \text {. }
$$

Proof: The results i) and ii) follows from Green's formula. $P\left(\frac{-2 k+1}{12}\right)=$ $Q\left(\frac{-2 k+1}{12}\right)=1$ imply that

$$
P(h)=\frac{1+o\left(h-\frac{-2 k+1}{12}\right)}{1+o\left(h-\frac{-2 k+1}{12}\right)}, \quad Q(h)=\frac{1+o\left(h-\frac{-2 k+1}{12}\right)}{1+o\left(h-\frac{-2 k+1}{12}\right)}
$$

as $h \rightarrow \frac{-2 k+1}{12}$. Noting $I_{i}(h)$ is analytic at $h=\frac{-2 k+1}{12}$ (see [15]) and $I_{0}(h)>0$ for $h \in\left(\frac{-2 k+1}{12}, 0\right)$, the formula $(2.15)$ implies that $P(h)$ and $Q(h)$ are analytic functions for $h \in\left[\frac{-2 k+1}{12}, 0\right)$.

Using

$$
P^{\prime}=\frac{I_{1}^{\prime} I_{0}-I_{0}^{\prime} I_{1}}{I_{0}^{2}}, \quad Q^{\prime}=\frac{I_{2}^{\prime} I_{0}-I_{0}^{\prime} I_{2}}{I_{0}^{2}}
$$

and system (2.2) give

$$
\left\{\begin{array}{l}
G P^{\prime}=a_{10}+\left(a_{11}-a_{00}\right) P+a_{12} Q-a_{01} P^{2}-a_{02} P Q \\
G Q^{\prime}=a_{20}+a_{21} P+\left(a_{22}-a_{00}\right) Q-a_{01} P Q-a_{02} Q^{2}
\end{array}\right.
$$

Differentiating (2.16) once (resp. twice) yields iii) (resp. iv)). [15])

It is well known that $I(h)$ has the expansion near $h=\frac{-2 k+1}{12}$ (see

$$
I(h)=b_{1}\left(h-\frac{-2 k+1}{12}\right)+b_{2}\left(h-\frac{-2 k+1}{12}\right)^{2}+\cdots .
$$




\section{Theorem 2.3.}

i)

$$
\begin{aligned}
& b_{1}=(\alpha+\beta+\gamma) I_{0}^{\prime}\left(\frac{-2 k+1}{12}\right), \\
& b_{2}=-\frac{(k-2) \beta+(k-3) \gamma}{2(k-1)^{2}} I_{0}^{\prime}\left(\frac{-2 k+1}{12}\right) \quad \text { if } b_{1}=0, \\
& b_{3}=\frac{5(k-2) \beta}{6(k-1)^{3}(k-3)} I_{0}^{\prime}\left(\frac{-2 k+1}{12}\right) \quad \text { if } b_{1}=b_{2}=0 .
\end{aligned}
$$

ii) If $b_{1}=0$ (resp. $\left.b_{1}=b_{2}=0\right), b_{2} \neq 0$ (resp. $\left.b_{3} \neq 0\right)$, then there exists one (resp. two) zero of $I(h)$ tend to $h=\frac{-2 k+1}{12}$, i.e., system $(1.8)_{\epsilon}$ has at most one (resp. two) limit cycle tend to $(1,0)$.

iii) The conditions $b_{1}=b_{2}=b_{3}=0$ hold if and only if $I(h) \equiv 0$.

Proof: (i) It follows from (1.7) and (2.14) that

$$
I(h)=I_{0}(h)(\alpha+\beta P(h)+\gamma Q(h)),
$$

which gives

$$
b_{m}=\left.\frac{1}{m !}\left\{\sum_{j=1}^{m}\left(\begin{array}{c}
m \\
j
\end{array}\right) I_{0}^{(m-j)}(h)[\alpha+\beta P(h)+\gamma Q(h)]^{(j)}\right\}\right|_{h=\frac{-2 k+1}{12}} .
$$

Therefore, the result i) follows from Proposition 2.2 and above equality.

(ii) In a neighbourhood of $(1,0)$, The Poincare map is

$$
P(h)=\epsilon I(h)+o(\epsilon)
$$

which yields ii).

(iii) The conditions $b_{1}=b_{2}=b_{3}=0$ hold if and only if

$$
\left\{\begin{array}{l}
\alpha+\beta+\gamma=0, \\
(k-2) \beta+(k-3) \gamma=0, \\
\beta=0,
\end{array}\right.
$$

which implies $\alpha=\beta=\gamma=0$. Hence, $I(h) \equiv 0$. 
Rewrite (1.5) in the form

$$
\frac{1}{2} y^{2}+\Phi(x)=h,
$$

where $\Phi(x)=-\frac{1}{2} k x^{2}+\frac{1}{3}(k+1) x^{3}-\frac{1}{4} x^{4}$ satisfying

$$
\Phi^{\prime}(x)(x-1)>0 \quad \text { for } \quad x \in(0,1) \cup\left(1, x_{1}\right) .
$$

For any $x \in(0,1)$, there is an unique $\widetilde{x} \in\left(1, x_{1}\right)$, such that

$$
\Phi(x)=\Phi(\widetilde{x}), \quad 0<x<1<\widetilde{x}<x_{1} .
$$

Therefore, we can define a function $\widetilde{x}=\widetilde{x}(x)$ for $0<x<1$ satisfying (2.22). By (2.21) and (2.22), we have

$$
\frac{d \widetilde{x}}{d x}=\frac{\Phi^{\prime}(x)}{\Phi^{\prime}(\widetilde{x})}<0 .
$$

Lemma 2.4. $x_{1}<x+\widetilde{x}<2, x \widetilde{x}<1$.

Proof: Let

$$
a=x+\widetilde{x}, \quad \text { and } \quad b=x \widetilde{x} .
$$

The equality (2.22) implies that

$$
\frac{1}{2} k a-\frac{1}{3}(k+1) a^{2}+\frac{1}{4} a^{3}+b\left[\frac{1}{3}(k+1)-\frac{1}{2} a\right]=0 .
$$

Taking $a=\frac{2}{3}(k+1)$ into $(2.25)$, we have $-\frac{1}{18}(2 k-1)(k-2)=0$, which contradicts the assumption $k>2$. This shows $a \neq \frac{2}{3}(k+1)$. Hence

$$
b=\frac{6 k a-4(k+1) a^{2}+3 a^{3}}{6 a-4(k+1)} .
$$

To find the maximal or minimal value of $a(x)$, we consider the equation $\frac{d a(x)}{d x}=0$, which is equivalent to

$$
\Phi^{\prime}(x)+\Phi^{\prime}(\widetilde{x})=0 .
$$

The relationship

$$
x^{2}+\widetilde{x}=a^{2}-2 b
$$

and (2.26) yield

$$
\Phi^{\prime}(x)+\Phi^{\prime}(\widetilde{x})=\frac{1}{2} a(a-2)(a-2 k) .
$$


The inequality $0<x<1<\widetilde{x}<k$ implies $0<a<2 k$, hence $a=2$ is the unique root of the equation (2.27). Noting $x \in[0,1]$ and $a(0)=x_{1}$, $a(1)=2$, we have

$$
x_{1}<a=x+\widetilde{x}<2,
$$

which implies that $x \widetilde{x}<x(2-x) \leq 1$.

Near the value $h=0$ corresponding to a saddle-loop $\Gamma_{0}$, Abelian integral $I(h)$ has the expansion $[\mathbf{1 4}]$

$$
I(h)=c_{0}+c_{1} h \ln |h|+c_{2} h+\cdots
$$

with $c_{0}=I(0), c_{1}=\left.\widetilde{c} \operatorname{div}(X, Y)\right|_{(0,0)}, c_{2}=I^{\prime}(0)$ if $c_{1}=0$, where $\widetilde{c}$ is a constant. Using this formula, we obtain

$$
\begin{aligned}
& I_{0}(h)=I_{0}(0)+c_{01} h \ln |h|+c_{02} h+\cdots, \\
& I_{1}(h)=I_{1}(0)+I_{1}^{\prime}(0) h+\cdots, \\
& I_{2}(h)=I_{2}(0)+I_{2}^{\prime}(0) h+\cdots .
\end{aligned}
$$

It follows from (1.7) and (2.30) that

$$
\begin{aligned}
& c_{0}=\alpha I_{0}(0)+\beta I_{1}(0)+\gamma I_{2}(0), \\
& c_{1}=\alpha c_{01}, \quad c_{01} \neq 0, \\
& c_{2}=\beta I_{1}^{\prime}(0)+\gamma I_{2}^{\prime}(0) \quad \text { if } \quad \alpha=0 .
\end{aligned}
$$

\section{Lemma 2.5.}

i) $\frac{d}{d h}\left(\frac{I_{2}(h)}{I_{1}(h)}\right)>0, h \in\left(\frac{-2 k+1}{12}, 0\right)$,

ii) $I_{1}(0) I_{2}^{\prime}(0)-I_{1}^{\prime}(0) I_{2}(0)>0$.

Proof: (i) Denote

$$
\xi(x)=\frac{x^{2}-\widetilde{x}^{2} \frac{\widetilde{d x}}{d x}}{x-\widetilde{x} \frac{d \widetilde{x}}{d x}}, \quad x \in(0,1) .
$$

It follows from (2.23) that

$$
\xi(x)=\frac{k-x \widetilde{x}}{k+1-x-\widetilde{x}},
$$

which gives

$$
\xi^{\prime}(x)=\frac{\mathcal{A}(x, \widetilde{x})}{(k+1-x-\widetilde{x})^{2}},
$$


where

$$
\begin{aligned}
\mathcal{A}(x, \widetilde{x}) & =-\left(\widetilde{x}+x \frac{d \widetilde{x}}{d x}\right)(k+1-x-\widetilde{x})+\left(1+\frac{d \widetilde{x}}{d x}\right)(k-x \widetilde{x}) \\
& =\frac{1}{\widetilde{x}(\widetilde{x}-1)(\widetilde{x}-k)}\left[\widetilde{x}(\widetilde{x}-1)^{2}(\widetilde{x}-k)^{2}+x(x-1)^{2}(x-k)^{2}\right] .
\end{aligned}
$$

Noting $0<x<1<\widetilde{x}<k$, we have $\mathcal{A}(x, \widetilde{x})<0$, which implies $\xi^{\prime}(x)<0$. Use this result and Theorem 1 of $[\mathbf{9}]$, we get i).

(ii) By symmetry and $\widetilde{x}=\widetilde{x}(x), \widetilde{z}=\widetilde{z}(z), y(\widetilde{x})=y(x), y(\widetilde{z})=y(z)$, we get

$$
\begin{aligned}
I_{1}(0) I_{2}^{\prime}(0) & -I_{1}^{\prime}(0) I_{2}(0) \\
& =2 \int_{0}^{x_{1}} x y d x \cdot 2 \int_{0}^{x_{1}} \frac{z^{2}}{y(z)} d z-2 \int_{0}^{x_{1}} \frac{z}{y(z)} d z \cdot 2 \int_{0}^{x_{1}} x^{2} y d x \\
& =2 \int_{0}^{x_{1}} \int_{0}^{x_{1}}\left[\frac{x y(x) z(z-x)}{y(z)}+\frac{x y(z) z(x-z)}{y(x)}\right] d x d z \\
& =2 \int_{0}^{1} \int_{0}^{1} \frac{\left[y^{2}(x)-y^{2}(z)\right]}{y(x) y(z)} \Phi(x, z) d x d z,
\end{aligned}
$$

where

$$
\begin{aligned}
\Phi(x, z) & =x z(z-x)-\widetilde{x} z(z-\widetilde{x}) \frac{d \widetilde{x}}{d x}-x \widetilde{z}(\widetilde{z}-x) \frac{d \widetilde{z}}{d z}+\widetilde{x} \widetilde{z}(\widetilde{z}-\widetilde{x}) \frac{d \widetilde{x}}{d x} \frac{d \widetilde{z}}{d z} \\
& =\left(x-\widetilde{x} \frac{d \widetilde{x}}{d x}\right)\left(z-\widetilde{z} \frac{d \widetilde{z}}{d z}\right)(\xi(z)-\xi(x)),
\end{aligned}
$$

$\xi(x)$ is defined as above. Since

$$
\xi^{\prime}(x)<0, \frac{d \widetilde{x}}{d x}<0, \frac{d \widetilde{z}}{d z}<0 \quad \text { and } \quad y^{\prime}(x)>0 \quad \text { for } \quad x \in(0,1)
$$

we get $I_{1}(0) I_{2}^{\prime}(0)-I_{1}^{\prime}(0) I_{1}(0)>0$.

Theorem 2.6. i) If $c_{0}=0, c_{1} \neq 0$ (resp. $\left.c_{0}=c_{1}=0\right)$, then $I(h)$ has at most one (resp. two) zero near $h=0$, i.e., system (1.8) $\epsilon$ has at most one (resp. two) limit cycle that tend to the saddle-loop $\Gamma_{0}$ of system (1.4).

ii) The condition $c_{0}=c_{1}=c_{2}=0$ is equivalent to $I(h) \equiv 0$. 
Proof: (i) It follows from Theorem $\mathrm{C}$ of $[\mathbf{1 4}]$.

(ii) Obviously, $c_{0}=c_{1}=c_{2}=0$ if and only if

$$
\left\{\begin{array}{l}
\alpha I_{0}(0)+\beta I_{1}(0)+\gamma I_{2}(0)=0 \\
\alpha=0 \\
\beta I_{1}^{\prime}(0)+\gamma I_{2}^{\prime}(0)=0
\end{array}\right.
$$

Lemma 2.5 implies that

$$
\left|\begin{array}{ccc}
I_{0}(0) & I_{1}(0) & I_{2}(0) \\
1 & 0 & 0 \\
0 & I_{1}^{\prime}(0) & I_{2}^{\prime}(0)
\end{array}\right|=-\left[I_{1}(0) I_{2}^{\prime}(0)-I_{1}^{\prime}(0) I_{2}(0)\right]<0 .
$$

Thus, $c_{0}=c_{1}=c_{2}=0$ if and only if $\alpha=\beta=\gamma=0$, which implies $I(h) \equiv 0$.

We end this section by several inequalities, which are crucial for our analysis in next two sections.

\section{Lemma 2.7.}

i) $I_{1}(0)<I_{2}(0)<I_{0}(0)$,

ii) $(k-2) I_{2}(0)-(k-3) I_{1}(0)-I_{0}(0)<0$.

Proof: (i) Lemma 2.5 i) and Proposition 2.2 imply that

$$
1=\frac{I_{2}\left(\frac{-2 k+1}{12}\right)}{I_{1}\left(\frac{-2 k+1}{12}\right)}<\frac{I_{2}(h)}{I_{1}(h)}<\frac{I_{2}(0)}{I_{1}(0)}
$$

for $h \in\left(\frac{-2 k+1}{12}, 0\right)$, which gives $I_{1}(0)<I_{2}(0)$. On the other hand,

$$
\begin{aligned}
I_{2}(0)-I_{0}(0) & =2 \int_{0}^{1}\left(x^{2}-1\right) y d x+2 \int_{1}^{x_{1}}(\widetilde{x}-1) y(\widetilde{x}) d \widetilde{x} \\
& =2 \int_{0}^{1} \frac{y}{\Phi^{\prime}(\widetilde{x})}(x-1)(\widetilde{x}-1)(\widetilde{x}-x)(k-x-\widetilde{x}-x \widetilde{x}) d x .
\end{aligned}
$$

It follows from Lemma 2.4 that $k-(x+\widetilde{x}+x \widetilde{x}) \geq k-3>0$. Hence, application of $(2.32)$ yields $I_{2}(0)<I_{0}(0)$. Summing up the above discussion, we get i).

(ii) Using same arguments as (i), we have

$$
\begin{aligned}
(k-2) & I_{2}(0)-(k-3) I_{1}(0)-I_{0}(0) \\
= & 2 \int_{0}^{1} \frac{y}{\Phi^{\prime}(\widetilde{x})}(x-1)(\widetilde{x}-1)(\widetilde{x}-x)[k-x-\widetilde{x}-(k-2) x \widetilde{x}] d x .
\end{aligned}
$$

It follows from Lemma 2.4 that $k-x-\widetilde{x}-(k-2) x \widetilde{x}>0$. Since $\Phi^{\prime}(x)>0$ and $0<x<1<\widetilde{x}$, the above equalities gives ii). 
3. Behaviour of curve $\omega(h)=\frac{I_{1}^{\prime \prime}(h)}{I_{0}^{\prime \prime}(h)}$ and relevant results

Lemma 3.1. For $h \in\left(\frac{-2 k+1}{12}, 0\right), I_{0}^{\prime \prime}(h)>0$.

Proof: Chow [3] and Gavrilov [6] have proved that the period function of (1.4) is monotonic, i.e., $I_{0}^{\prime \prime}(h) \neq 0$ for $h \in\left(\frac{-2 k+1}{12}, 0\right)$. On the other hand, since $I_{0}^{\prime}(h)>0$, the formula $(2.30)$ implies $c_{01}<0$. This gives

$$
I_{0}^{\prime \prime}(h)=\frac{c_{01}}{h}+\cdots>0
$$

as $h \rightarrow 0^{-}$, which yields the result.

Define

$$
\omega(h)=\frac{I_{1}^{\prime \prime}(h)}{I_{0}^{\prime \prime}(h)}, \quad h \in\left(\frac{-2 k+1}{12}, 0\right) .
$$

In this section, we shall derive the Riccati equation satisfied by $\omega(h)$ and discuss the behaviour of curve $\omega(h)$. The upshot is to prove that $I(h)$ has at most three zeros in $\left(\frac{-2 k+1}{12}, 0\right)$.

\section{Lemma 3.2.}

$$
\begin{aligned}
I_{2}^{\prime \prime}(h)=\frac{-12 h}{(2 k-1)(k-2)} & I_{0}^{\prime \prime}(h) \\
& +\frac{1}{k+1}\left[\frac{36 h}{(k-2)(2 k-1)}+k\right] I_{1}^{\prime \prime}(h) .
\end{aligned}
$$

Proof: Differentiating both sides of (2.1) yields

$$
(4 h \mathbf{E}+\mathbf{S}) \mathbf{J}^{\prime \prime}=(\mathbf{N}-4 \mathbf{E}) \mathbf{J}^{\prime},
$$

where

$$
\mathbf{N}-4 \mathbf{E}=\left(\begin{array}{ccc}
-1 & 0 & 0 \\
-\frac{1}{3}(k+1) & 0 & 0 \\
\frac{1}{3}\left(-k^{2}+k-1\right) & -\frac{2}{3}(k+1) & 1
\end{array}\right) .
$$

Eliminating $I_{0}^{\prime}$ from the first two equations of (3.3), we get (3.2). 
Lemma 3.3. The integral $I_{0}, I_{1}$ satisfy the following equation

$$
G(h)\left(\begin{array}{l}
I_{0}^{\prime \prime \prime} \\
I_{1}^{\prime \prime \prime}
\end{array}\right)=\left(\begin{array}{ll}
A(h) & B(h) \\
C(h) & D(h)
\end{array}\right)\left(\begin{array}{l}
I_{0}^{\prime \prime} \\
I_{1}^{\prime \prime}
\end{array}\right)
$$

where

$$
\begin{aligned}
A(h)= & \frac{48\left(-7 k^{2}+22 k-7\right)}{(2 k-1)(k-2)} h^{2}+\frac{4}{3}\left(14 k^{4}-27 k^{3}-10 k^{2}-27 k+14\right) h \\
& +\frac{4}{3} k^{3}(2 k-1)(k-2), \\
B(h)= & \frac{1}{k+1}\left[\frac{-432\left(k^{2}-k+1\right)}{(2 k-1)(k-2)} h^{2}+\frac{4}{3}\left(-2 k^{4}+k^{3}+60 k^{2}+k-2\right) h\right. \\
& \left.-\frac{1}{9} k^{2}(2 k-1)(k-2)\left(10 k^{2}+11 k+10\right)\right], \\
C(h)= & \frac{16(k+1)\left(7 k^{2}-13 k+7\right)}{(2 k-1)(k-2)} h^{2}+\frac{4}{3} k(k+1)(2 k-1)(k-2) h, \\
D(h)= & \frac{-48\left(17 k^{2}-38 k+17\right)}{(2 k-1)(k-2)} h^{2}+\frac{4}{3}\left(10 k^{4}-21 k^{3}+10 k^{2}-21 k+10\right) h .
\end{aligned}
$$

Proof: Differentiate once (3.3), we get

$$
(4 h \mathbf{E}+\mathbf{S}) \mathbf{J}^{\prime \prime \prime}=(\mathbf{N}-8 \mathbf{E}) \mathbf{J}^{\prime \prime}
$$

Substituting (3.2) into the first two equations of (3.5), we get (3.4).

Theorem 3.4. The ratio $\omega(h)$ satisfies the following Riccati equation

$$
G(h) \omega^{\prime}(h)=C(h)+(D(h)-A(h)) \omega-B(h) \omega^{2} .
$$

Proof: Since

$$
\omega^{\prime}=\frac{I_{1}^{\prime \prime \prime} I_{0}^{\prime \prime}-I_{1}^{\prime \prime} I_{0}^{\prime \prime \prime}}{\left(I_{0}^{\prime \prime}\right)^{2}}
$$

the equation (3.6) follows from Lemma 3.3.

Lemma 3.5. For $h \in\left(\frac{-2 k+1}{12}, 0\right), B(h)<0, C(h)<0$, which implies $(D(h)-A(h))^{2}+4 B(h) C(h)>0$. 
Proof: Denote

$$
\begin{aligned}
B_{1}(h)= & \frac{4}{3}\left(-2 k^{4}+k^{3}+60 k^{2}+k-2\right) h \\
& -\frac{1}{9} k^{2}(2 k-1)(k-2)\left(10 k^{2}+11 k+10\right), \\
C_{1}(h)= & \frac{16(k+1)\left(7 k^{2}-13 k+7\right)}{(2 k-1)(k-2)} h+\frac{4}{3} k(k+1)(2 k-1)(k-2),
\end{aligned}
$$

which gives

$$
B(h)=\frac{1}{k+1}\left[\frac{-432\left(k^{2}-k+1\right)}{(2 k-1)(k-2)} h^{2}+B_{1}(h)\right], \quad C(h)=h C_{1}(h) .
$$

Since $B_{1}(h)$ is linear function of $h$ and

$$
\begin{aligned}
B_{1}(0) & =-\frac{1}{9} k^{2}(2 k-1)(k-2)\left(10 k^{2}+11 k+10\right)<0 \\
B_{1}\left(\frac{-2 k+1}{12}\right) & =-\frac{1}{9}(2 k-1)\left[( k - 2 ) \left(10 k^{4}+9 k^{3}\right.\right. \\
& \left.\left.+7 k^{2}+54 k+109\right)+216\right]<0
\end{aligned}
$$

this shows $B_{1}(h)<0$. It follows from $(3.7)$ that $B(h)<0$.

Similarly, we get $C_{1}(h)>0$, which implies $C(h)<0$ for $h \in\left(\frac{-2 k+1}{12}, 0\right)$.

Proposition 3.6. For $h \in\left(\frac{-2 k+1}{12}, 0\right), \omega(h)$ is analytic and

i) $\omega^{\prime}(h)>0$,

ii) $-\frac{(k+1)(2 k-7)}{10 k^{2}-31 k+31}<\omega(h)<0$.

Proof: By Theorem 3.4, the curve $\omega(h)$ is the trajectory of system

$$
\left\{\begin{array}{l}
\dot{h}=G(h), \\
\dot{\omega}=C(h)+(D(h)-A(h)) \omega-B(h) \omega^{2},
\end{array}\right.
$$

which has four critical points in $\left\{(h, \omega) \mid \frac{-2 k+1}{12} \leq h \leq 0\right\}$ : a stable node at $E_{1}(0,0)$, two saddles at $J_{1}\left(\frac{-2 k+1}{12}, \frac{(k+1)(2 k-7)}{10 k^{2}-31 k+31}\right)$ and $E_{2}\left(0, \frac{12 k(k+1)}{10 k^{2}+11 k+10}\right)$, an unstable node at $J_{2}\left(\frac{-2 k+1}{12}, 1\right)$. The isocline $\omega^{ \pm}(h)$ is determined by algebraic curve

$$
C(h)+(D(h)-A(h)) \omega-B(h) \omega^{2}(h)=0,
$$


where

$$
\begin{aligned}
\omega^{+}(h) & =\frac{D-A-\sqrt{(D-A)^{2}+4 B C}}{2 B}, \\
\omega^{-}(h) & =\frac{D-A+\sqrt{(D-A)^{2}+4 B C}}{2 B}
\end{aligned}
$$

with

$$
\omega^{-}\left(\frac{-2 k+1}{12}\right)=-\frac{(k+1)(2 k-7)}{10 k^{2}-31 k+31}, \quad \omega^{-}(0)=0 .
$$

Differentiating (3.9) once, we have

$$
\begin{aligned}
& \left(\omega^{-}\right)^{\prime}\left(\frac{-2 k+1}{12}\right) \\
& \quad=\frac{35(k-2)(k+1)(2 k-1)\left(2 k^{2}-11 k+11\right)}{(k-1)^{2}\left(10 k^{2}-31 k+31\right)^{2}}>0 .
\end{aligned}
$$

Assume $\frac{d \omega^{-}}{d h}=0$ at $h=\bar{h}$ and $\left(\omega^{-}\right)^{\prime}(h)>0$ for $h \in\left(\frac{-2 k+1}{12}, \bar{h}\right)$, which implies $\left(\omega^{-}\right)^{\prime \prime}(\bar{h})<0$. Differentiate $(3.9)$ twice to get

$$
\left(\omega^{-}\right)^{\prime \prime}(\bar{h})=\frac{432\left(k^{2}-k+1\right)\left(\omega^{-}-\frac{k+1}{3}\right)\left(\omega^{-}-\frac{(k+1)\left(7 k^{2}-13 k+7\right)}{9\left(k^{2}-k+1\right)}\right)}{B(h)(k+1)(2 k-1)(k-2)\left(\omega^{-}-\frac{D-A}{2 B}\right)} .
$$

By Lemma 3.5 and (3.11), we have $\omega^{-}-\frac{D-A}{2 B}<0, B(\bar{h})<0$ and $\omega^{-}(\bar{h})<0$. Therefore, the formula $(3.13)$ gives $\left(\omega^{-}\right)^{\prime \prime}(\bar{h})>0$. This contradicts the assumption, which yields that the isocline $\omega=\omega^{-}(h)$ is monotonically increasing function for $h \in\left(\frac{-2 k+1}{12}, 0\right)$.

Since $I_{i}(h)$ is analytic at $h=\frac{-2 k+1}{12}$, it follows from (3.4) that

$$
A\left(\frac{-2 k+1}{12}\right) I_{0}^{\prime \prime}\left(\frac{-2 k+1}{12}\right)+B\left(\frac{-2 k+1}{12}\right) I_{2}^{\prime \prime}\left(\frac{-2 k+1}{12}\right)=0,
$$

which implies

$$
\omega\left(\frac{-2 k+1}{12}\right)=-\frac{(k+1)(2 k-7)}{10 k^{2}-31 k+31} .
$$

Lemma 3.1 and (3.14) show that $\omega(h)$ is analytic for $h \in\left[\frac{-2 k+1}{12}, 0\right)$. On the other hand, the formula (2.30) gives

$$
\omega(0)=\lim _{h \rightarrow 0} \frac{I_{1}^{\prime \prime}}{I_{0}^{\prime \prime}}=0 .
$$


Hence, $\omega(h)$ is the trajectory of (3.13) from $J_{1}$ to $E_{1}$. Since $\left(\omega^{-}\right)^{\prime}(h)>0$, the graph of $\omega(h)=\frac{I_{1}^{\prime \prime}}{I_{0}^{\prime \prime}}$ must stay in the region $\left\{(h, \omega) \mid \omega<\omega^{-}, h \in\right.$ $\left.\left(\frac{-2 k+1}{12}, 0\right)\right\}$, which implies $\omega^{\prime}(h)>0$, see Figure 3.1. The inequality ii) follows from i), (3.14) and (3.15).

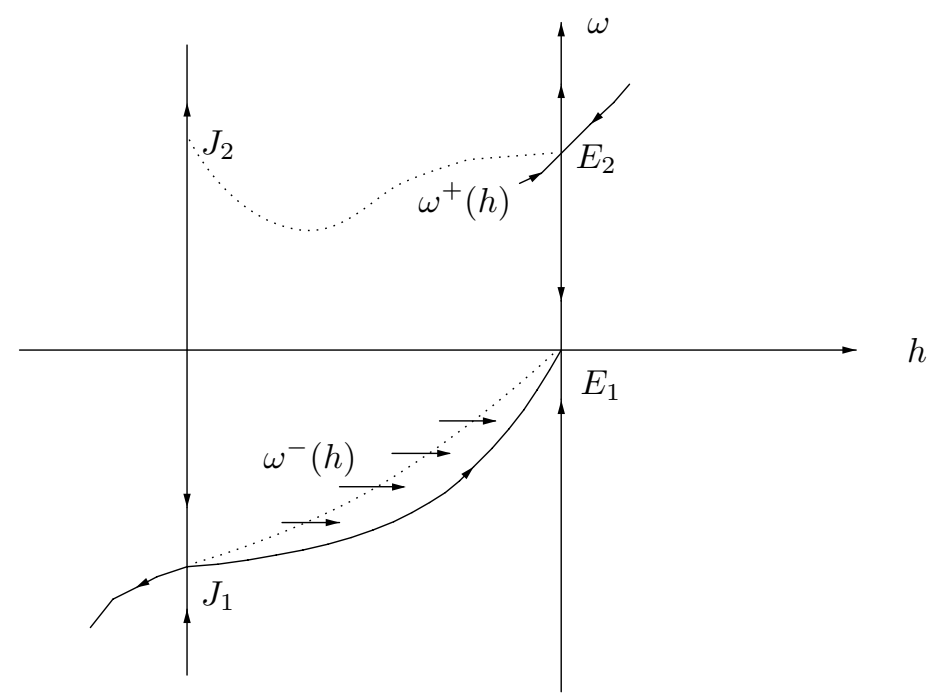

Figure 3.1

Corollary 3.7. $\quad$ i) If $\alpha+\frac{1}{3}(\gamma k+\beta k+\beta)=0, \gamma \neq 0$, then $I^{\prime \prime}(h)$ has $h=h^{*}=-\frac{(\gamma k+\beta k+\beta)(k-2)(2 k-1)}{36 \gamma}$ as the unique zero in $\left(\frac{-2 k+1}{12}, 0\right)$. If $\alpha+\frac{1}{3}(\gamma k+\beta k+\beta) \neq 0$, then $h=h^{*}$ is not the zero of $I^{\prime \prime}(h)$.

ii) If $\alpha+\frac{1}{3}(\gamma k+\beta k+\beta)>0,-\frac{k-2}{k-3} \beta<\gamma<0$, then $I^{\prime \prime}(h)$ has at most one zero in $\left(\frac{-2 k+1}{12}, 0\right)$.

iii) $P^{\prime}(h)<0$ for $h \in\left(\frac{-2 k+1}{12}, 0\right)$.

Proof: (i) Lemma 3.2 yields

$$
\begin{aligned}
I^{\prime \prime}(h)=\frac{(2 k-1)(k-2) \alpha-12 \gamma h}{(2 k-1)(k-2)} I_{0}^{\prime \prime} \\
\quad+\frac{36 \gamma h+(\gamma k+\beta k+\beta)(2 k-1)(k-2)}{(k+1)(2 k-1)(k-2)} I_{1}^{\prime \prime} .
\end{aligned}
$$


If $\alpha+\frac{1}{3}(\gamma k+\beta k+\beta)=0$, then

$$
I^{\prime \prime}(h)=\frac{36 \gamma I_{0}^{\prime \prime}(h)\left(\omega(h)-\frac{k+1}{3}\right)\left(h-h^{*}\right)}{(k+1)(2 k-1)(k-2)},
$$

which implies that $I^{\prime \prime}(h)$ has $h=h^{*}$ as the unique zero. If $\alpha+\frac{1}{3}(\gamma k+$ $\beta k+\beta) \neq 0$, then it follows from Lemma 3.1 and (3.16) that

$$
I^{\prime \prime}\left(h^{*}\right)=\left[\alpha+\frac{1}{3}(\gamma k+\beta k+\beta)\right] I_{0}^{\prime \prime}\left(h^{*}\right) \neq 0 .
$$

(ii) In the case of $\alpha+\frac{1}{3}(\gamma k+\beta k+\beta)>0,-\frac{k-2}{k-3} \beta<\gamma<0, h=h^{*}$ is not a zero of $I^{\prime \prime}(h)$, and (3.16) is equivalent to

$$
I^{\prime \prime}(h)=\frac{36 \gamma\left(h-h^{*}\right)}{(k+1)(2 k-1)(k-2)} I_{0}^{\prime \prime}(h) q(h),
$$

where

$$
q(h)=f(h)+\omega(h)
$$

and

$$
f(h)=\frac{(k+1)[(2 k-1)(k-2) \alpha-12 \gamma h]}{36 \gamma\left(h-h^{*}\right)},
$$

which implies

$$
f^{\prime}(h)=-\frac{(k+1)(2 k-1)(k-2)\left[\alpha+\frac{1}{3}(\gamma k+\beta k+\beta)\right]}{36 \gamma\left(h-h^{*}\right)^{2}}>0 .
$$

Therefore, by Proposition 3.6, we have

$$
q^{\prime}(h)=f^{\prime}(h)+\omega^{\prime}(h)>0 .
$$

If $-\frac{k+1}{k} \beta \leq \gamma<0$, then $h^{*} \geq 0$. This and (3.20) imply that $q(h)$ (i.e., $\left.I^{\prime \prime}(h)\right)$ has at most one zero in $h \in\left(\frac{-2 k+1}{12}, 0\right)$.

On the other hand, if $-\frac{k-2}{k-3} \beta<\gamma<-\frac{k+1}{k} \beta<0$, then $h^{*} \in\left(\frac{-2 k+1}{12}, 0\right)$, $\alpha>-\frac{1}{3}(\gamma k+\beta k+\beta)$. The inequality (3.19) gives

$$
f(h)<f(0)=\frac{(k+1) \alpha}{\gamma k+\beta k+\beta}<0
$$

for $h \in\left(h^{*}, 0\right)$. Hence, Proposition 3.6 yields $q(h)<0$ for $h^{*}<h<0$. It follows from $(3.20)$ that $q(h)$ has at most one zero in $h \in\left(\frac{-2 k+1}{12}, h^{*}\right)$. We obtain ii) by using i), Lemma 3.1 and (3.17). 
(iii) Consider the Abelian integral

$$
I(h)=\alpha I_{0}(h)+I_{1}(h)=I_{0}(h)(\alpha+P(h)) .
$$

If $\alpha>0$, then $I(h)>0$.

If $\alpha<0$, then Lemma 3.1 and Proposition 3.6 show that

$$
I^{\prime \prime}(h)=I_{0}^{\prime \prime}(h)(\alpha+\omega(h))<0,
$$

which implies the curve $I(h)$ is concave for $h \in\left(\frac{-2 k+1}{12}, 0\right)$. Therefore, noticing $I\left(\frac{-2 k+1}{12}\right)=0$ and $I_{0}(h) \neq 0, \alpha+P(h)$ has at most one zero for arbitrary constant $\alpha$. This yields $P(h)$ is monotonic for $h \in\left(\frac{-2 k+1}{12}, 0\right)$. Suppose $h=h_{1}$ is the zero of $I(h)$, the convexity of $I(h)$ implies $I^{\prime}\left(h_{1}\right)=$ $I_{0}\left(h_{1}\right) P^{\prime}\left(h_{1}\right) \leq 0$, i.e. $P^{\prime}\left(h_{1}\right) \leq 0$. However, if $P^{\prime}\left(h_{1}\right)=0$, then

$$
\begin{aligned}
I^{\prime \prime}\left(h_{1}\right) & =I_{0}^{\prime \prime}\left(h_{1}\right)\left(\alpha+P\left(h_{1}\right)\right)+2 I_{0}^{\prime}\left(h_{1}\right) P^{\prime}\left(h_{1}\right)+I_{0}\left(h_{1}\right) P^{\prime \prime}\left(h_{1}\right) \\
& =I_{0}\left(h_{1}\right) P^{\prime \prime}\left(h_{1}\right)<0,
\end{aligned}
$$

which shows $P^{\prime \prime}\left(h_{1}\right)<0$, i.e., $h=h_{1}$ is the maximum point of $P(h)$. This contradicts $P^{\prime}\left(h_{1}\right) \leq 0$. The proof is finished.

Proposition 3.8. $\omega^{\prime \prime}(h)>0$ for $h \in\left(\frac{-2 k+1}{12}, 0\right)$.

Proof: We split the proof by several steps.

1) First, $V(h, \omega)=2 D^{\prime}-2 A^{\prime}-G^{\prime \prime}-4 B^{\prime} \omega>0$.

It is readily seen

$$
\begin{gathered}
V(h, 0)=-\frac{384}{(2 k-1)(k-2)}[(k-2)(11 k-1)+9] h \\
\quad+\frac{16}{3}\left(4 k^{2}-k+4\right)(k-1)^{2}>0 \\
V\left(h,-\frac{(2 k-7)(k+1)}{10 k^{2}-31 k+31}\right)=-\frac{384\left(55 k^{2}-139 k+139\right) h}{10 k^{2}-31 k+31} \\
\quad+\frac{16}{3\left(10 k^{2}-31 k+31\right)}\left[k^{3}(k-4)\left(40 k^{2}-58 k+287\right)\right. \\
\left.+582 k^{3}+\left(211 k^{2}-414 k\right)+138\right]>0
\end{gathered}
$$

Since $V(h, \omega)$ is linear function of $\omega$ and $\omega^{\prime}(h)>0,-\frac{(k+1)(2 k-7)}{10 k^{2}-31 k+31}<$ $\omega<0$ (see Proposition 3.6), it follows from (3.21) that $V(h, \omega)>0$ for $h \in\left(\frac{-2 k+1}{12}, 0\right)$. 
2) If $h=h_{1}$ satisfies $\omega^{\prime \prime}\left(h_{1}\right)=0$, then $\omega^{\prime \prime \prime}\left(h_{1}\right)>0$.

Indeed, differentiate (3.6) twice to get

$$
\begin{array}{r}
G\left(h_{1}\right) \omega^{\prime \prime \prime}\left(h_{1}\right)=C^{\prime \prime}\left(h_{1}\right)+\left(D^{\prime \prime}\left(h_{1}\right)-A^{\prime \prime}\left(h_{1}\right)\right) \omega\left(h_{1}\right)-B^{\prime \prime} \omega^{2}\left(h_{1}\right) \\
+V\left(h_{1}, \omega\left(h_{1}\right)\right) \omega^{\prime}\left(h_{1}\right)-2 B\left(h_{1}\right)\left(\omega^{\prime}\right)^{2}\left(h_{1}\right) .
\end{array}
$$

By Lemma 3.5, Proposition 3.6 and step 1),we conclude that

$$
\begin{gathered}
C^{\prime \prime}\left(h_{1}\right)+\left(D^{\prime \prime}\left(h_{1}\right)-A^{\prime \prime}\left(h_{1}\right)\right) \omega\left(h_{1}\right)-B^{\prime \prime}\left(h_{1}\right) \omega^{2}\left(h_{1}\right) \\
=\frac{864\left(k^{2}-k+1\right)}{(k+1)(2 k-1)(k-2)}\left(\omega\left(h_{1}\right)-\frac{k+1}{3}\right) \\
\times\left(\omega\left(h_{1}\right)-\frac{(k+1)\left(7 k^{2}-13 k+7\right)}{9\left(k^{2}-k+1\right)}\right)>0, \\
\quad V\left(h_{1}, \omega\left(h_{1}\right)\right) \omega^{\prime}\left(h_{1}\right)>0, \quad-2 B\left(h_{1}\right)\left(\omega^{\prime}\right)^{2}\left(h_{1}\right)>0 .
\end{gathered}
$$

Hence, the formulas (3.22) and (3.23) imply $\omega^{\prime \prime \prime}\left(h_{1}\right)>0$.

3) $\omega^{\prime \prime}\left(\frac{-2 k+1}{12}\right)>0$.

To prove it, differentiating (3.6) twice, we get

$$
\omega^{\prime \prime}\left(\frac{-2 k+1}{12}\right)=\frac{35(k-2)(k+1)(2 k-1)}{72(k-1)^{5}\left(10 k^{2}-31 k+31\right)^{3}} g(k),
$$

where

$$
\begin{aligned}
g(k)=2200 k^{6}-24924 k^{5}+129246 k^{4}- & 375481 k^{3} \\
+ & 604833 k^{2}-500511 k+166837 .
\end{aligned}
$$

This gives $g^{(i)}(4)>0, i=0,1,2, \ldots, 6$, which implies

$$
g(k)=\sum_{i=0}^{6} \frac{g^{(i)}(4)}{i !}(k-4)^{i}>0, \quad k \in(4,+\infty) .
$$

Hence, the result $\omega^{\prime \prime}\left(\frac{-2 k+1}{12}\right)>0$ follows from (3.24) and (3.25).

4) Finally, we prove $\omega^{\prime \prime}(h)>0$.

By step 3), starting from $h=\frac{-2 k+1}{12}$, if $h=h_{1}$ is the first point satisfying $\omega^{\prime \prime}\left(h_{1}\right)=0$, then $\omega^{\prime \prime \prime}\left(h_{1}\right) \leq 0$, which contradicts the result proved in step 2). This implies that $\omega^{\prime \prime}(h)$ has no zero. Therefore, $\omega^{\prime \prime}(h)>0$.

Theorem 3.9. $I(h)$ has at most three zeros (counted with their multiplicities) inside the interval $\left(\frac{-2 k+1}{12}, 0\right)$. 
Proof: This theorem is proved by several parts.

1) We are going to prove that $I^{\prime \prime}(h)$ has at most two zeros (counted with their multiplicities), i.e., $I(h)$ has at most two inflection points. Since $I\left(\frac{-2 k+1}{12}\right)=0$, this result implies that the maximum number of zeros of $I(h)$ is at most three on the interval $\left(\frac{-2 k+1}{12}, 0\right)$.

It has been proved in Proposition 3.6 that $\omega^{\prime}(h)>0$. Therefore, we can take $\omega$ as a new parameter and consider the curve $\nu=\nu(h(\omega))$, defined by

$$
\widetilde{\Omega}=\left\{(\omega, \nu) \mid \omega=\omega(h), \nu=\nu(h)=\frac{I_{2}^{\prime \prime}}{I_{0}^{\prime \prime}}, h \in\left(\frac{-2 k+1}{12}, 0\right)\right\}
$$

where $h=h(\omega)$ is the inverse function of $\omega=\omega(h)$. It is easy to get that

$$
\begin{aligned}
& I^{\prime \prime}(h)=I_{0}^{\prime \prime}(h)(\alpha+\beta \omega(h)+\gamma \nu(h)), \\
& I^{\prime \prime \prime}(h)=I_{0}^{\prime \prime}(h)\left(\beta \omega^{\prime}+\gamma \nu^{\prime}\right) \quad \text { if } I^{\prime \prime}(h)=0, \\
& I^{(4)}(h)=I_{0}^{\prime \prime}(h)\left(\beta \omega^{\prime \prime}+\gamma \nu^{\prime \prime}\right) \quad \text { if } \quad I^{\prime \prime}(h)=I^{\prime \prime \prime}(h)=0,
\end{aligned}
$$

which implies that $\widetilde{\Omega}$ has the following properties:

i) The intersection points of the lines $l: \alpha+\beta \omega+\gamma \nu=0$ with the curve $\widetilde{\Omega}$ in $\omega \nu$-plane correspond to the zeros of $I^{\prime \prime}(h)$.

ii) $I^{\prime \prime}\left(h_{0}\right)=I^{\prime \prime \prime}\left(h_{0}\right)=0$ hold if and only if $l$ is tangent to the $\widetilde{\Omega}$ at the point $\left(\omega\left(h_{0}\right), \nu\left(h_{0}\right)\right)$.

iii) If $\left.\left(\nu^{\prime \prime} \omega^{\prime}-\nu^{\prime} \gamma^{\prime \prime}\right)\right|_{h=h_{0}} \neq 0$, then $I^{\prime \prime}\left(h_{0}\right)=I^{\prime \prime \prime}\left(h_{0}\right)=I^{(4)}\left(h_{0}\right)=0$ hold if and only if $\alpha=\beta=\gamma=0$, i.e, $I(h) \equiv 0$.

Lemma 3.2 gives

$$
\nu(h)=-\frac{12 h}{(2 k-1)(k-2)}+\left[\frac{36 h}{(k+1)(2 k-1)(k-2)}+\frac{k}{k+1}\right] \omega,
$$

which yields

$$
\nu^{\prime \prime} \omega^{\prime}-\omega^{\prime \prime} \nu^{\prime}=\frac{12}{(k+1)(k-2)(2 k-1)}\left[6\left(\omega^{\prime}\right)^{2}+(k+1-3 \omega) \omega^{\prime \prime}\right]
$$

It follows from Proposition 3.6, Proposition 3.8 and (3.27) that

$$
\frac{d^{2} \nu}{d \omega^{2}}=\frac{\nu^{\prime \prime} \omega^{\prime}-\omega^{\prime \prime} \nu^{\prime}}{\left(\omega^{\prime}\right)^{3}}>0
$$

This implies that $\widetilde{\Omega}$ is convex in $\omega \nu$-plane. Therefore, the maximum number of intersection points of the line $l: \alpha+\beta \omega+\gamma \nu=0$ with $\widetilde{\Omega}$ is at most two. By the properties i)-iii) of $\widetilde{\Omega}, I^{\prime \prime}(h)$ has at most two zeros (counted with their multiplicities). 
2) The multiplicity of zero of $I(h)$ is at most three. If $h=h_{0}$ is the zero of multiplicity 3 , then $h=h_{0}$ is an unique zero of $I(h)$.

Otherwise, suppose the multiplicity of $h=h_{0}$ is great than 3, i.e., $I\left(h_{0}\right)=I^{\prime}\left(h_{0}\right)=I^{\prime \prime}\left(h_{0}\right)=I^{\prime \prime \prime}\left(h_{0}\right)$. By step 1$), I^{\prime \prime}(h)$ has at most two zeros (counted with their multiplicities) in $h \in\left(\frac{-2 k+1}{12}, 0\right)$, which implies $I^{(4)}\left(h_{0}\right) \neq 0$. Without loss of generality, assume $I^{(4)}(h)>0$. Hence, $I(h)$ is convex in the neighbourhood of $h=h_{0}$. Noting $I\left(\frac{-2 k+1}{12}\right)=0$, there must exist one inflection point $h=h_{1}, h_{1} \in\left(\frac{-2 k+1}{12}, h_{0}\right)$, see Figure 3.2(a). Thus, $I^{\prime \prime}(h)$ has two zeros, one is simple and another is multiplicity two. This contradicts the conclusion proved in step 1$)$.

Suppose $h=h_{0}$ is the zero with multiplicity 3, i.e., $I\left(h_{0}\right)=I^{\prime}\left(h_{0}\right)=$ $I^{\prime \prime}\left(h_{0}\right)=0, I^{\prime \prime \prime}\left(h_{0}\right) \neq 0$. Without loss of generality, assume $I^{\prime \prime \prime}\left(h_{0}\right)>0$. Hence, the graph of $I(h)$ is convex for $h>h_{0}$ and concave for $h<h_{0}$, $\left|h-h_{0}\right| \ll 0$. Since $I\left(\frac{-2 k+1}{12}\right)=0, I(h)$ has another inflection point $h=$ $h_{1}$ between $h=\frac{-2 k+1}{12}$ and $h=h_{0}$, see Figure 3.2(b). By step 1), $I(h)$ has no other inflection point except $h=h_{i}, i=0,1$, which implies $h=h_{0}$ is an unique zero of $I(h)$.

3) If $h=h_{0}$ is the zero of multiplicity two of $I(h)$, then another zero $h=h_{1}$ (if there exists) must be simple.

Obviously, $h=h_{0}$ satisfies $I\left(h_{0}\right)=I^{\prime}\left(h_{0}\right)=0, I^{\prime \prime}\left(h_{0}\right) \neq 0$. Without loss of generality, suppose $I^{\prime \prime}\left(h_{0}\right)>0$, i.e., $h=h_{0}$ is minimal point of $I(h)$. Suppose $h_{1}>h_{0}$. Then there must exist two inflection points between $\frac{-2 k+1}{12}$ and $h_{1}$. Hence, it follows from step 1) that $h=h_{1}$ must be simple zero of $I(h)$. In the case of $h_{1}<h_{0}$, we can get the result by the same arguments as above.

Summing up above discussion, we get the theorem.

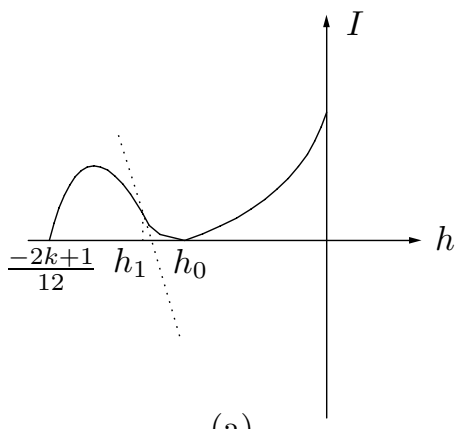

(a)

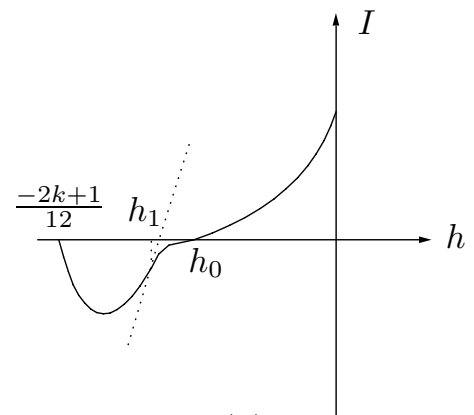

(b)

Figure 3.2 


\section{The geometry of the centriod curve}

Definition 4.1. In $P Q$-plane, the curve

$$
\Omega=\left\{(P, Q) \mid P=P(h), \quad Q=Q(h), \quad h \in\left[\frac{-2 k+1}{12}, 0\right]\right\}
$$

is called centroid curve.

It has been proved in Corollary 3.7 that $P^{\prime}(h)<0$. Therefore, $P$ can be taken as a new parameter and denote $\Omega$ as

$$
Q=Q(h(p))
$$

where $h(P)$ is the inverse function of $P=P(h)$.

The importance of concept of the centroid curve lies in the fact that its geometry contains the complete information of $I(h)$ although the definition of $\Omega$ depends only on $H(x, y)=h$.

From this section, denoted by $L_{s}$ and by $L_{c}$ the tangents to $\Omega$ at $(P(0), Q(0))$ and $(1,1)$, i.e., at the endpoints of $\Omega$. $L$ denotes the line $\alpha+$ $\beta P+\gamma Q=0,|\beta|+|\gamma| \neq 0$.

Using same arguments as [4], we have

Theorem 4.2. $\quad$ i) For any $h_{0} \in\left(\frac{-2 k+1}{12}, 0\right)$, the equality $I\left(h_{0}\right)=0$ holds if and only if the line $L$ passes through the point $\left(P\left(h_{0}\right), Q\left(h_{0}\right)\right)$.

ii) The equalities $I\left(h_{0}\right)=I^{\prime}\left(h_{0}\right)=0$ hold if and only if $L$ is tangent to the centroid curve $\Omega$ at the point $\left(P\left(h_{0}\right), Q\left(h_{0}\right)\right)$.

iii) If $I\left(h_{0}\right)=I^{\prime}\left(h_{0}\right)=0$, then $I^{\prime \prime}\left(h_{0}\right)=0$ holds if and only if $P^{\prime}\left(h_{0}\right) Q^{\prime \prime}\left(h_{0}\right)-P^{\prime \prime}\left(h_{0}\right) Q^{\prime}\left(h_{0}\right)=0$, i.e., the curvature of $\Omega$ at $\left(P\left(h_{0}\right), Q\left(h_{0}\right)\right)$ is zero.

Proof: (i) Part i) of the statement follows from (2.19).

(ii) The equation of the tangent line is

$$
Q^{\prime}\left(h_{0}\right) P-P^{\prime}\left(h_{0}\right) Q+Q\left(h_{0}\right) P^{\prime}\left(h_{0}\right)-Q^{\prime}\left(h_{0}\right) P\left(h_{0}\right)=0 .
$$

By $(2.19), I\left(h_{0}\right)=I^{\prime}\left(h_{0}\right)=0$ is equivalent to

$$
\left\{\begin{array}{l}
\alpha+\beta P\left(h_{0}\right)+\gamma Q\left(h_{0}\right)=0, \\
\beta P^{\prime}\left(h_{0}\right)+\gamma Q^{\prime}\left(h_{0}\right)=0 .
\end{array}\right.
$$


Solving (4.3) for $\alpha$ and $\beta$, we obtain that

$$
\alpha=\frac{P\left(h_{0}\right) Q^{\prime}\left(h_{0}\right)-P^{\prime}\left(h_{0}\right) Q\left(h_{0}\right)}{P^{\prime}\left(h_{0}\right)} \gamma, \quad \beta=-\frac{Q^{\prime}\left(h_{0}\right)}{P^{\prime}\left(h_{0}\right)} \gamma .
$$

Hence, the equation $L: \alpha+\beta P+\gamma Q=0$ is (4.2).

(iii) The condition $I^{\prime \prime}\left(h_{0}\right)=0$ when $I\left(h_{0}\right)=I^{\prime}\left(h_{0}\right)=0$ is equivalent to

$$
\beta P^{\prime \prime}\left(h_{0}\right)+\gamma Q^{\prime \prime}\left(h_{0}\right)=0 .
$$

This and (4.3) imply the result.

Theorem 4.3. $\quad$ i) The equation of $L_{c}$ is

$$
-1-(k-3) P+(k-2) Q=0 .
$$

ii) The coefficient $b_{1}=0$ if and only if $L$ passes through $(1,1)$.

iii) The conditions $b_{1}=b_{2}=0$ hold if and only if $L=L_{c}$, where $b_{1}$ and $b_{2}$ are defined as Theorem 2.3.

Proof: (i) Part i) of the statement follows from Proposition 2.2.

(ii) By Theorem 2.3, $b_{1}=I_{0}^{\prime}\left(\frac{-2 k+1}{12}\right)(\alpha+\beta+\gamma)$, which implies ii).

(iii) Theorem 2.3 shows that $b_{1}=b_{2}=0$ if and only if

$$
\left\{\begin{array}{l}
\alpha+\beta+\gamma=0 \\
(k-2) \beta+(k-3) \gamma=0 .
\end{array}\right.
$$

Solving this system for $\alpha$ and $\beta$, we obtain $\alpha=-\frac{1}{k-2} \gamma, \beta=-\frac{k-3}{k-2} \gamma$, which implies that the equation of $L$ is (4.4).

Theorem 4.4. $\quad$ i) The equation of $L_{s}$ is

$$
\frac{Q}{P}=\frac{Q(0)}{P(0)}
$$

ii) The coefficient $c_{0}$ is zero if and only if $L$ passes through $(P(0), Q(0))$.

iii) The coefficient $c_{0}=c_{1}=0$ is equivalent to $L=L_{s}$, where $c_{0}, c_{1}$ is defined as (2.31).

Proof: (i) By (2.30) and Lemma 3.1, $\lim _{h \rightarrow 0} I_{0}^{\prime}(h)=+\infty, \lim _{h \rightarrow 0} I_{1}^{\prime}(h)=$ $I_{1}^{\prime}(0), \lim _{h \rightarrow 0} I_{2}^{\prime}(h)=I_{2}^{\prime}(0), \lim _{h \rightarrow 0} I_{i}(h)=I_{i}(0), i=0,1,2$. Therefore,

$\left.\frac{d Q}{d P}\right|_{h=0}=\left.\frac{d Q}{d h} \frac{d h}{d P}\right|_{h=0}=\lim _{h \rightarrow 0} \frac{I_{2}^{\prime} I_{0}-I_{0}^{\prime} I_{2}}{I_{1}^{\prime} I_{0}-I_{0}^{\prime} I_{1}}=\lim _{h \rightarrow 0} \frac{\frac{I_{2}^{\prime} I_{0}}{I_{0}^{\prime}}-I_{2}}{\frac{I_{1}^{\prime} I_{0}}{I_{0}^{\prime}}-I_{1}}=\frac{Q(0)}{P(0)}$,

which yields that the equation of $L_{s}$ is (4.5). 
(ii) By (2.31), the condition $c_{0}=0$ is equivalent to $\alpha+\beta P(0)+$ $\gamma Q(0)=0$, which implies ii).

(iii) It follows from (2.31) that $c_{0}=c_{1}=0$ if and only if

$$
\left\{\begin{array}{l}
\alpha+\beta P(0)+\gamma Q(0)=0, \\
\alpha=0
\end{array}\right.
$$

which implies $\alpha=0, \beta=-\frac{Q(0)}{P(0)} \gamma$. Therefore, the equation of $L$ is (4.5). The result follows.

Proposition 4.5. $L_{c s}$ doesn't intersect $\Omega$ for $h \in\left(\frac{-2 k+1}{12}, 0\right)$, where $L_{c s}$ is the line passing through both $(1,1)$ and $(P(0), Q(0))$.

Proof: By the definition of $L_{c s}$ and Theorem 4.3, Theorem 4.4, we have

$$
\left\{\begin{array}{l}
\alpha+\beta+\gamma=0 \\
\alpha I_{0}(0)+\beta I_{1}(0)+\gamma I_{2}(0)=0
\end{array}\right.
$$

which implies

$$
\alpha=\frac{I_{1}(0)-I_{2}(0)}{I_{2}(0)-I_{0}(0)} \beta, \quad \gamma=\frac{I_{0}(0)-I_{1}(0)}{I_{2}(0)-I_{0}(0)} \beta .
$$

If $\beta=0$, then $\gamma=0$, which contradicts the assumption $|\beta|+|\gamma| \neq$ 0 . Without loss of generality, suppose $\beta>0$. The formula (4.6) and Lemma 2.7 give that $\gamma<0$ and

$$
\begin{aligned}
& \alpha+\frac{1}{3}(\gamma k+\beta k+\beta)=\frac{\beta}{3\left[I_{2}(0)-I_{1}(0)\right]} \\
& \gamma+\frac{k-2}{k-3} \beta=\frac{\left[(k-2) I_{2}(0)-(k-3) I_{1}(0)-I_{0}(0)\right]>0,}{(k-3)\left[I_{2}(0)-I_{0}(0)\right]} \\
& {\left[(k-2) I_{2}(0)-(k-3) I_{1}(0)-I_{0}(0)\right]>0 . }
\end{aligned}
$$

Corollary 3.7 yields that $I(h)$ has at most one inflection point. Since $I(0)=I\left(\frac{-2 k+1}{12}\right)=I^{\prime}\left(\frac{-2 k+1}{12}\right)=0($ cf. Theorem 2.3), $I(h)$ has no zero in $\left(\frac{-2 k+1}{12}, 0\right)$. The result follows from Theorem 4.2 . 
Proposition 4.6. i) $L_{s}$ doesn't intersect $\Omega$ for $h \in\left[\frac{-2 k+1}{12}, 0\right)$.

ii) $L_{c}$ doesn't intersect $\Omega$ except the endpoint $(1,1)$.

iii) The centroid curve $\Omega$ is concave near its endpoints $(1,1)$ and $(P(0), Q(0))$.

Proof: (i) Denoted by $Q(h)$ and by $Q$ the ordinates of the points on $\Omega$ and $L_{s}$ respectively. By Theorem 4.4 i), we have

$$
Q(h)-Q=Q(h)-\frac{Q(0)}{P(0)} P(h)=P(h)\left[\frac{I_{2}(h)}{I_{1}(h)}-\frac{I_{2}(0)}{I_{1}(0)}\right] .
$$

Lemma 2.5 implies that $\frac{I_{2}(h)}{I_{1}(h)}<\frac{I_{2}(0)}{I_{1}(0)}$ for $h \in\left(\frac{-2 k+1}{12}, 0\right)$, which yields $Q(h)<Q$, i.e., $L_{s}$ doesn't intersect $\Omega$ except $h=0$.

(ii) By Theorem 4.3 iii), $L_{c}$ is tangent to $\Omega$ at $(1,1)$ if and only if $b_{1}=b_{2}=0$, i.e., $\gamma=-\frac{k-2}{k-3}, \alpha=\frac{\beta}{k-3}$. This gives $\alpha+\frac{1}{3}(\gamma k+\beta k+$ $\beta)=0$. It follows from Corollary 3.7 that $h=h^{*}=\frac{-2 k+1}{12}$ is an unique zero of $I^{\prime \prime}(h)$, which shows that $I(h)$ has no inflection point for $h \in\left(\frac{-2 k+1}{12}, 0\right)$. Since the curve $I(h)$ is tangent to $h$-axis at $h=\frac{-2 k+1}{12}$ (cf. Theorem 2.3 and Theorem 4.3) and $I\left(\frac{-2 k+1}{12}\right)=0, I(h)$ has no zero in the interval $\left(\frac{-2 k+1}{12}, 0\right)$. By Theorem $4.2, L_{c}$ does not intersect $\Omega$ for $h \in\left(\frac{-2 k+1}{12}, 0\right)$. Since Proposition 4.5 shows that $L_{c}$ doesn't pass through $(P(0), Q(0))$, we get ii).

(iii) Proposition 2.2 gives

$$
\left.\frac{d^{2} Q}{d P^{2}}\right|_{(1,1)}=\left.\frac{Q^{\prime \prime} P^{\prime}-P^{\prime \prime} Q^{\prime}}{\left(P^{\prime}\right)^{3}}\right|_{h=\frac{-2 k+1}{12}}=-\frac{20(k-1)}{3(k-2)^{2}}<0,
$$

which shows that $\Omega$ is concave near the endpoint $(1,1)$.

From (2.30), near $h=0$, we have

$$
\frac{d^{2} Q}{d P^{2}}=\frac{1}{\left(P^{\prime}(h)\right)^{3}}\left\{\frac{c_{01}}{h I_{0}^{3}(0)}\left(I_{1}(0) I_{2}^{\prime}(0)-I_{1}^{\prime}(0) I_{2}(0)+o\left(h^{-1}\right)\right\} .\right.
$$

In the proof of Lemma 3.1, one gets $c_{01}<0$. It follows from Lemma 2.5 and Lemma 2.7 iii) that $\frac{d^{2} Q}{d P^{2}}<0$ as $h \rightarrow 0^{-}$, i.e., $\Omega$ is cancave near the endpoint $(P(0), Q(0))$. 
The analysis we have done shows that

Corollary 4.7. The centriod curve $\Omega$ is entirely placed in the triangle formed by $L_{s}, L_{c}$ and $L_{c s}$, see Figure 4.1.

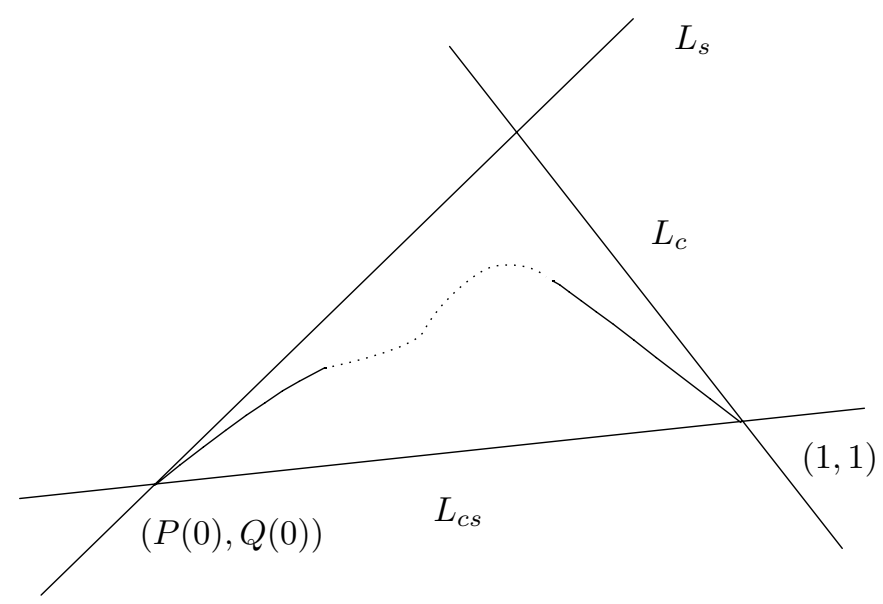

Figure 4.1

\section{Proof of main theorem}

Theorem 4.2-4.4 reduce the proof of Theorem 1.1 to showing that each line $L: \alpha+\beta P+\gamma Q=0$ intersects the centriod curve $\Omega$ in at most two points, which implies $\Omega$ is a strictly concave curve.

As a sequence of Theorem 3.9 and Theorem 4.2, the following assertion holds:

Lemma 5.1. If the line $L$ does not pass through $(1,1)$ or $(P(0), Q(0))$, then $L$ intersects $\Omega$ in at most three points (counted with their multiplicities).

Lemma 5.2. Each line $L$ intersects the centriod curve $\Omega$ in at most two points (counted with their multiplicities).

Proof: We split the proof in several steps.

1) Each line $L$, passing through $(1,1)$ or $(P(0), Q(0))$, intersects $\Omega$ in at most two points (counted with their multiplicities).

For $L=L_{c}, L_{s}$ or $L_{c s}$, we have proved the conclusion in Proposition 4.5 and Proposition 4.6. Suppose now that $L$ is a line through $(1,1), L \neq L_{c}, L \neq L_{c s}$, which has another common point $M$ with $\Omega$, 
$M \neq(1,1)$ and $(P(0), Q(0))$. Then obviously the points of $\Omega$ near $(1,1)$ and those near $(P(0), Q(0))$ lie on different side of $L$ (cf. Corollary 4.7), which yields that either $L$ has no other common point with $\Omega$ than $(1,1)$ and $M$ ( $M$ is simple), or the total number of intersection points is at least 3 except $(1,1)$ (see Figure $5.1(\mathrm{a})$ ). Now we prove the latter case is impossible.

Indeed, by the conclusion proved in step 1) of the proof of Theorem 3.9, we know that $I(h)$ has at most two inflection points in $\left(\frac{-2 k+1}{12}, 0\right)$. Since $I\left(\frac{-2 k+1}{12}\right)=I^{\prime}\left(\frac{-2 k+1}{12}\right)=0$ when $L$ passes through $(1,1)$ (cf. Theorem 2.3 and Theorem 4.3), $I(h)$ has at most two zeros except $h=\frac{-2 k+1}{12}$, see Figure 5.1(b), i.e., $L$ has at most two common points with $\Omega$ except $(1,1)$, which contradicts the latter case.

If $L$ is a line through $(P(0), Q(0))$ and $L \neq L_{s}, L \neq L_{c s}$, then $I\left(\frac{-2 k+1}{12}\right)=I(0)=0$. Using the result proved in step 1) of the proof of Theorem 3.9 again, we have that $I(h)$ has at most two zeros except $h=0$ and $h=\frac{-2 k+1}{12}$. Using the same arguments as above, we get that $L$ intersects $\Omega$ in at most two points including $(P(0), Q(0))$.

2) Each tangent $L(h), h \in\left(\frac{-2 k+1}{12}, 0\right)$, to $\Omega$ at point $(P(h), Q(h))$ has exactly one common double point with $\Omega$ (the point of tangence).

Indeed, starting from $(P(0), Q(0))$, suppose that $M_{0}=\left(P\left(h_{0}\right), Q\left(h_{0}\right)\right)$, $h_{0} \in\left(\frac{-2 k+1}{12}, 0\right)$ is the first point for which $L\left(h_{0}\right)$ has another common point $M_{1}$ with $\Omega$ (i.e., $M_{1}=\left(P\left(h_{1}\right), Q\left(h_{1}\right), h \neq h_{0}\right)$. By the result proved in step 1), $M_{1}$ doesn't coincide with $(1,1)$ and $(P(0), Q(0))$. The choice of $M_{1}$ being the first such point implies $L\left(h_{0}\right)$ is tangent to $\Omega$ also at $M_{1}$ (see Figure 5.2), which contradicts Lemma 5.1. Consequently, there is no $h_{1} \in\left[\frac{-2 k+1}{12}, 0\right]$, for which $L\left(h_{0}\right)$ has another common point with $\Omega$ except the tangency point. To prove that is a double intersection point, assume the contrary. Then by Lemma 5.1 the point $\left(P\left(h_{0}\right), Q\left(h_{0}\right)\right)$ is a triple point of intersection. Theorem 4.2 yields $I\left(h_{0}\right)=I^{\prime}\left(h_{0}\right)=$ $I^{\prime \prime}\left(h_{0}\right)=0$. Slightly moving the tangent $L\left(h_{0}\right)$, we find suitable $h_{1}, h_{2}$ near $h_{0}$, for which $I\left(h_{1}\right)=I^{\prime}\left(h_{1}\right)=0, I\left(h_{2}\right)=0$. Then accordingly to Theorem 4.2, $L\left(h_{1}\right)$ is tangent to $\Omega$ at $\left(P\left(h_{1}\right), Q\left(h_{1}\right)\right)$, which intersects $\Omega$ in another point $\left(P\left(h_{2}\right), Q\left(h_{2}\right)\right)$. This contradicts the fact we proved above.

3) Suppose that $L$ is not a tangent to $\Omega$ at any point, $L \neq L_{c}, L_{s}$ and $L_{c s}$. By step 2), $\Omega$ is placed entirely on one side of each of its tangents, otherwise the number of the intersection points would be at least 3 . This implies $\Omega$ is strictly concave. Therefore, $L$ intersects $\Omega$ in at most two simple points. Lemma is proved. 


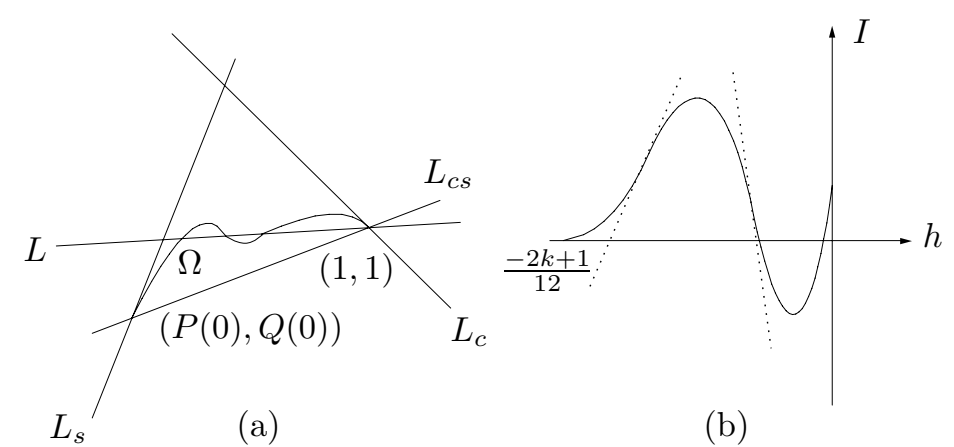

Figure 5.1

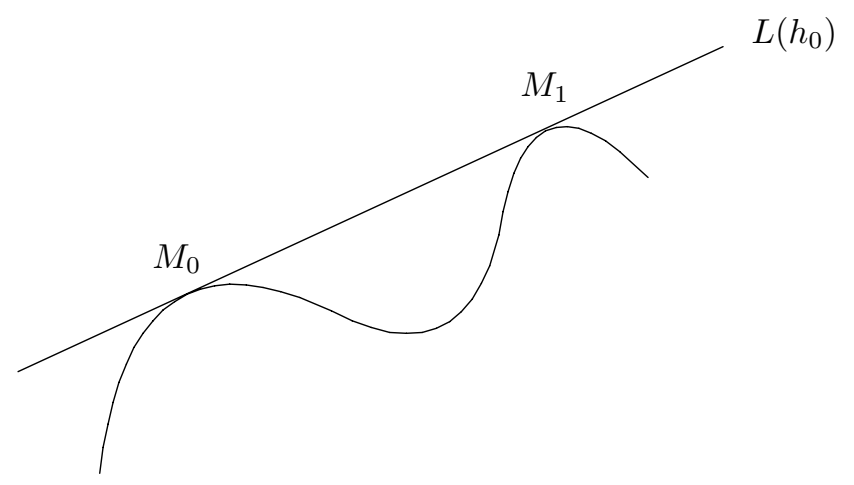

Figure 5.2

Proof of Theorem 1.1: For a given perturbation $(1.8)_{\epsilon}$, if $\beta=\gamma=0$, then either the divergenve in $(1.8)_{\epsilon}$ vanishes identically or it is nowhere zero. In the first case, $(1.8)_{\epsilon}$ is a Hamiltonian system and in second one no limit cycle can appear in (1.8) $)_{\epsilon}$. Suppose $|\beta|+|\gamma| \neq 0$, which means that the line $L$ is defined. By Lemma 5.2, Theorem 2.3, Theorem 2.6 and Theorem 4.2-4.4, the theorem follows.

\section{References}

[1] V. I. ARNold, "Geometrical methods in the theory of ordinary differential equation", Springer Verlag/Berlin/Heidelberg/New York, 1988. 
[2] V. I. Arnold, Ten problem, in: "Theory of singularities and its applications", Adv. Soviet Math. 1, Amer. Math. Soc., Providence, R.I., 1990, pp. 1-8.

[3] S. N. Chow And J. A. Sanders, On the number of critical points of period, J. Differential Equations 64(1) (1986), 51-66.

[4] E. Horozov And I. D. Iliev, On the number of limit cycles in perturbation of quadratic Hamiltonian systems, Proc. London Math. Soc. (3) 69(1) (1994), 198-224.

[5] J. M. Jebrane And H. ZoladeK, Abelian integrals in nonsymmetric perturbation of symmetric Hamiltonian vector field, Adv. in Appl. Math. 15(1) (1994), 1-12.

[6] L. Gavrilov, Remark on the number of critical points of the period, J. Differential Equations 101(1) (1993), 58-65.

[7] B. Li AND Z. Zhang, A note on a result of G. S. Petrov about the weakened 16th Hilbert problem, J. Math. Anal. Appl. 190(2) (1995), 489-516.

[8] C. Li, J. Llibre And Z. Zhang, Abelian integrals of quadratic Hamiltonian vector fields with an invariant straight line, Publ. Mat. 39(2) (1995), 355-366.

[9] C. Li And Z. Zhang, A criterion for determining the monotonicity of the ratio of two Abelian integrals, J. Differential Equations 124(2) (1996), 407-424.

[10] G. S. Petrov, The number of zeros of complete elliptic integrals, Funktsional. Anal. i Prilozhen. 18(2) (1984), 73-74 (Russian).

[11] G. S. Petrov, The Chebyshev property of elliptic integrals, Funktsional. Anal. i Prilozhen. 22(1) (1988), 83-84 (Russian); translation in Functional Anal. Appl. 22(1) (1988), 72-73.

[12] G. S. Petrov, Complex zeroes of an elliptic integral, Funktsional. Anal. i Prilozhen. 23(2) (1989), 88-89 (Russian); translation in Functional Anal. Appl. 23(2) (1989), 160-161.

[13] G. S. Petrov, Complex zeros of an elliptic integral, Funktsional. Anal. i Prilozhen. 21(3) (1987), 87-88.

[14] R. Roussarie, On the number of limit cycles which appear by perturbation of separatrix loop of planar vector fields, Bol. Soc. Brasil. Mat. 17(2) (1986), 67-101.

[15] R. Roussarie, "Bifurcation of planar vector fields and Hilbert's sixteenth problem", Progress in Mathematics 164, Birkhäuser Verlag, Basel, 1998.

[16] Y. Zhao And Z. Zhang, Abelian integrals for cubic vector fields, Ann. Mat. Pura Appl. (4) CLXXVI (1999), 251-272. 
[17] Y. ZhaO AND Z. ZHANG, Linear estimate of the number of zeros of abelian integrals for a kind of quartic Hamiltonians, J. Differential Equations 155(1) (1999), 73-88.

Yulin Zhao:

Department of Mathematics

Zhongshan University

Guangzhou 510275

People's Republic of China

E-mail address: mcszyl@zsu.edu.cn

Zhifen Zhang:

Department of Mathematics

Peking University

Beijing 100871

People's Republic of China

E-mail address: zhangzf@sxx0.math.pku.edu.cn

Primera versió rebuda el 25 de març de 1999, darrera versió rebuda el 24 de febrer de 2000. 